\title{
Spin transport and magnetoresistance in ferromagnet/superconductor/ferromagnet spin valves
}

\author{
Jan Petter Morten, ${ }^{1, *}$ Arne Brataas, ${ }^{1}$ and Wolfgang Belzig ${ }^{2}$ \\ ${ }^{1}$ Department of Physics, Norwegian University of Science and Technology, 7491 Trondheim, Norway \\ ${ }^{2}$ Department of Physics and Astronomy, University of Basel, Klingelbergstrasse 82, 4056 Basel, Switzerland
}

(Received 25 January 2005; revised manuscript received 7 April 2005; published 6 July 2005)

\begin{abstract}
We consider spin transport and spin relaxation in superconductors using the quasiclassical theory of superconductivity. We include spin relaxation due to spin-orbit interaction as well as magnetic impurities, and show that the energy dependence of the spin-flip rate is different for these two mechanisms. In ferromagnetsuperconductor-ferromagnet systems made of $\mathrm{Co}$ and $\mathrm{Al}$, interface resistances can be small compared to bulk resistances. This simplifies the description of transport in $\mathrm{Co} / \mathrm{Al} / \mathrm{Co}$ spin valves, for which we numerically calculate the temperature and $\mathrm{Al}$ length dependence of the magnetoresistance.
\end{abstract}

PACS number(s): 74.25.Fy, 72.25.Hg, 72.25.Ba

\section{INTRODUCTION}

Ferromagnetism and superconductivity are two competing phenomena in condensed matter physics. In conventional low temperature superconductors, transport of spins beyond the coherence length is prevented by the formation of spin singlet Cooper pairs. Consequently, due to the competing ordering of ferromagnets $(\mathrm{F})$ and superconductors $(\mathrm{S})$ in hybrid structures, many nontrivial physical effects occur ${ }^{1,2}$ and there are interesting suggested applications such as an absolute spin valve effect ${ }^{3}$ and solid state memory elements. ${ }^{4}$

Experimental studies of $\mathrm{F} / \mathrm{S}$ contacts in the diffusive limit showed that the resistance can both decrease and increase relative to the resistance above the critical temperature $\left(T_{c}\right)$ of the superconductor. ${ }^{5-7}$ Theoretically it was shown that the temperature dependence of this resistance depends sensitively on the contact transparency. ${ }^{8-10}$ The resulting resistance is determined by an interplay between the energydependent interface resistance and spin accumulation at the interfaces due to reduced spin transport into the superconductor.

Transport of spins through the bulk of superconductors was recently studied experimentally in an $\mathrm{F} / \mathrm{S} / \mathrm{F}$ heterostructure. ${ }^{11}$ Here, a decreased magnetoresistance (MR) in the superconducting state was interpreted as a loss of spin memory. Theoretical work on bulk spin transport in superconductors in the inelastic regime ${ }^{12}$ and the elastic regime ${ }^{13}$ describes the reduced penetration of spins by spin flipping and reduced penetration of spin-polarized quasiparticles. The F/S/F system of Ref. 11 has been analyzed by assuming a spatially homogeneous superconducting order parameter and neglecting spin flip. ${ }^{14}$ However, a thorough understanding of spin transport in $\mathrm{F} / \mathrm{S}$ systems requires a description of the spatially dependent order parameter in each component as well as the quasiparticles driven out of equilibrium. A theoretical description of the F/S/F system, where the spatial variation of the order parameter, energy-dependent spin flipping in the superconductor, and the effect of the interfaces is taken into account, has to the best of our knowledge not been published.

In order to study the bulk spin-transport properties, it is important to have control over the influence of interfaces.
Typically, in spin valve structures both interface resistances and bulk resistivities contribute to the MR and are affected by superconductivity. In this paper, we study a superconducting spin valve system, where the interface resistances are negligible. In that case, a simplified treatment of the F/S boundaries is possible so that bulk effects can be studied independently of interface effects. As we discuss later, a possible candidate to realize a spin valve with small interface resistance could be a $\mathrm{Co} / \mathrm{Al} / \mathrm{Co}$ system. To describe the transport through a superconducting spin valve, we present a theoretical framework that describes the spin-dependent transport in superconductors in linear response. Spin-flip scattering from magnetic impurities as well as spin-orbit interaction is included in our description, and the full spatial dependence of the pairing potential is calculated selfconsistently. We use this formalism for numerical calculations of the magnetization-configuration dependent transport of a $\mathrm{Co} / \mathrm{Al} / \mathrm{Co}$ spin valve. This demonstrates the suppression of spin transport through the superconductor.

The paper is organized in the following way: Section II describes the equations governing elastic transport in a diffusive superconductor. Section III outlines the specific geometry studied and the approximations used. In Sec. IV we discuss the numerical results. Section V summarizes and concludes our work.

\section{TRANSPORT THEORY}

Using the Keldysh theory in the quasiclassical approximation, we have in Ref. 13 derived kinetic equations for transport of charge, energy, spin, and spin energy in diffusive, $s$-wave superconductors in the presence of spin-flip scattering by magnetic impurities. We will now supplement that treatment with expressions for spin-orbit induced spin relaxation, and derive the resulting transport equations in the linear response limit. For an explanation of the notations used below and more details on the derivations we refer to Ref. 13.

The spin-orbit interaction Hamiltonian is

$$
H_{\mathrm{so}}=\frac{\gamma}{2} \sum_{\sigma^{\prime} \sigma} \int d \mathbf{r} \psi_{\sigma^{\prime}}^{\dagger}\left\{\left(\overline{\boldsymbol{\tau}} \times \nabla V_{\mathrm{imp}}\right)_{\sigma^{\prime} \sigma} \cdot \mathbf{p}+\text { H.c. }\right\} \psi_{\sigma},
$$

where $\gamma$ is the interaction strength, $\psi_{\sigma}$ is the destruction field operator for spin $\sigma=\uparrow, \downarrow, \bar{\tau}$ is the vector of Pauli matrices, 
$V_{\text {imp }}$ is the impurity scattering potential and $\mathbf{p}$ the momentum. The spin-orbit contribution to the self-energy in the Eilenberger equation ${ }^{15}$ is

$$
\check{\sigma}_{\mathrm{so}}=-\frac{i}{2 \tau_{\mathrm{so}}} \frac{1}{4} \hat{\boldsymbol{\alpha}} \hat{\rho}_{3} \check{g}_{s}(X, E) \hat{\rho}_{3} \hat{\boldsymbol{\alpha}},
$$

where we have defined the spin-orbit scattering time $1 / \tau_{\text {so }}$ $=8 \gamma^{2} p_{F}^{4} / 9 \tau$. Here $p_{F}$ is the Fermi momentum, $\tau$ is the elastic scattering time, $\hat{\boldsymbol{\alpha}}$ is a vector of $4 \times 4$ matrices with the Pauli matrix and its transpose on the diagonal block, i.e., $\hat{\boldsymbol{\alpha}}$ $=\operatorname{diag}\left(\overline{\boldsymbol{\tau}}, \overline{\boldsymbol{\tau}}^{T}\right), \hat{\rho}_{3}=\operatorname{diag}(1,1,-1,-1)$, and $\check{g}_{s}$ is the isotropic part of the Green's function in Keldysh-Nambu-spin-space. Using a convenient representation of the Green's functions, we obtain equations that determine the distribution functions and currents.

Linearized kinetic equations for charge transport in diffusive superconductors were obtained by Schmid and Schön ${ }^{16}$ and have been successfully applied to describe various transport phenomena. To study spin-dependent transport it is necessary to include equations that determine the spin current. The relevant equations in the linear response regime are developed below. The approximations are valid when deviations from equilibrium values are small. We also assume that any static supercurrent is small, i.e., that there is no Josephson effect. The transport theory is formulated in terms of the physical particle and energy currents (including particles and holes). These are given by the distribution functions $h_{\mathrm{T}}$ and $h_{\mathrm{L}}$ (Ref. 17) and the spin-resolved functions $h_{\mathrm{TS}}$ and $h_{\mathrm{LS}}$, as well as generalized diffusion coefficients $D_{\mathrm{T}}, D_{\mathrm{L}}$ and renormalization factors $\alpha_{\mathrm{TT}}, \alpha_{\mathrm{TSTS}}$ for relaxation processes. The spin-resolved distribution functions can be expressed by the particle distribution functions $f_{\uparrow}$ and $f_{\downarrow}$ as

$$
\underset{\mathrm{LS}}{h_{\mathrm{TS}}}=-\frac{f_{\uparrow}(E)-f_{\downarrow}(E)}{2} \mp \frac{f_{\uparrow}(-E)-f_{\downarrow}(-E)}{2} .
$$

The spectral (retarded) properties depend on the complex function $\theta(E)$ which is determined by the so-called Usadel equation. ${ }^{17}$ To describe spin-polarized transport in voltage biased systems in linear response, it is unnecessary to calculate $h_{\mathrm{L}}$ and $h_{\mathrm{LS}}$, so the equations that determine these functions have been omitted below.

The charge current and the spin current in S are given by integrals over the spectral quantities. The charge and spin current carried by quasiparticles is ${ }^{29}$

$$
\begin{gathered}
I_{\text {charge }}^{\mathrm{qp}}(x)=\frac{1}{2} e A N_{0} \int_{-\infty}^{\infty} d E D_{\mathrm{T}}(E, x) \frac{\partial h_{\mathrm{T}}}{\partial x}, \\
I_{\text {spin }}(x)=\frac{1}{2} e A N_{0} \int_{-\infty}^{\infty} d E D_{\mathrm{L}}(E, x) \frac{\partial h_{\mathrm{TS}}}{\partial x},
\end{gathered}
$$

where $A$ is the area of the wire and $N_{0}$ is the density of states at the Fermi level for both spins in the normal state. Additionally, charge current is carried by the supercurrent, so that the total charge current is constant. The distribution functions $h_{\mathrm{T}}$ and $h_{\mathrm{TS}}$ are determined by the diffusion equations

$$
\begin{gathered}
\frac{\partial}{\partial x}\left(D_{\mathrm{T}} \frac{\partial h_{\mathrm{T}}}{\partial x}\right)-2 \Delta \alpha_{\mathrm{TT}} h_{\mathrm{T}}=0 \\
\frac{\partial}{\partial x}\left(D_{\mathrm{L}} \frac{\partial h_{\mathrm{TS}}}{\partial x}\right)-\left(\frac{1}{\tau_{\mathrm{m}}} \alpha_{\mathrm{TSTS}}^{\mathrm{m}}+\frac{1}{\tau_{\mathrm{so}}} \alpha_{\mathrm{TSTS}}^{\mathrm{so}}\right) h_{\mathrm{TS}}=0 .
\end{gathered}
$$

Here $\tau_{\mathrm{m}}$ is the spin-flip scattering time due to magnetic impurities and $\tau_{\mathrm{so}}$ the spin-flip scattering time due to spin-orbit coupling, both evaluated in the normal state. In the diffusive limit $\tau \ll \tau_{\mathrm{m}}, \tau_{\mathrm{so}}$. The spectral quantities are given in terms of $\theta(E, x)$. We compute that the renormalization of the scattering rates are

$$
\begin{gathered}
\alpha_{\mathrm{TT}}=\operatorname{Im}\{\sinh (\theta)\}, \\
\alpha_{\mathrm{TSTS}}^{\mathrm{so}}=(\operatorname{Re}\{\cosh (\theta)\})^{2}-(\operatorname{Re}\{\sinh (\theta)\})^{2}, \\
\alpha_{\mathrm{TSTS}}^{\mathrm{m}}=(\operatorname{Re}\{\cosh (\theta)\})^{2}+(\operatorname{Re}\{\sinh (\theta)\})^{2}, \\
D_{\mathrm{L}}=D\left[(\operatorname{Re}\{\cosh (\theta)\})^{2}-(\operatorname{Re}\{\sinh (\theta)\})^{2}\right], \\
D_{\mathrm{T}}=D\left[(\operatorname{Re}\{\cosh (\theta)\})^{2}+(\operatorname{Im}\{\sinh (\theta)\})^{2}\right] .
\end{gathered}
$$

The effect of spin-flip scattering by spin-orbit interaction with renormalization factor $\alpha_{\text {TSTS }}^{\text {so }}$ is a result that did not appear in our previous paper. ${ }^{13}$ Its renormalization is different from the renormalization of the spin-flip scattering by magnetic impurities. The complex function $\theta$ is determined by the Usadel equation,

$$
\hbar D \frac{\partial^{2} \theta}{\partial x^{2}}=-2 i \Delta \cosh (\theta)-2 i E \sinh (\theta)+\frac{3}{4} \frac{\hbar}{\tau_{\mathrm{m}}} \sinh (2 \theta) .
$$

Note that the spin-flip term in (9) arises from magnetic impurities only since spin-orbit scattering does not lead to pair breaking and consequently does not influence the spectral properties of the superconductor. This equation must be solved in conjunction with the self-consistency relation

$$
\Delta=\frac{1}{2} N_{0} \lambda \int_{0}^{E_{\mathrm{D}}} d E \operatorname{Re}\{\sinh (\theta)\} \tanh \left(\frac{\beta E}{2}\right),
$$

where $\lambda$ is the electron-electron interaction strength, $E_{\mathrm{D}}$ the Debye cutoff energy, and $\beta$ the inverse temperature.

An applied voltage is taken into account as a boundary condition for the distribution functions, $h_{\mathrm{T}}$ and $h_{\mathrm{TS}}$. In a reservoir with electrochemical potential $\mu$ we have in linear response the equilibrium distributions $h_{\mathrm{T}}^{0}$ $=-\beta \mu /\left[2 \cosh ^{2}(\beta E / 2)\right]$ and $h_{\mathrm{TS}}^{0}=0$.

The different renormalization factors $\alpha_{\text {TSTS }}^{\text {so }}$ and $\alpha_{\text {TSTS }}^{\mathrm{m}}$ arise from spin flipping by spin-orbit interaction or magnetic impurities. In general, $\alpha_{\text {TSTS }}^{\text {so }}$ and $\alpha_{\text {TSTS }}^{\mathrm{m}}$ depend on the spectral properties of the superconductor through $\theta$. In the BCS limit, valid for large bulk superconductors, the energy dependence of these factors is completely different and correspond to the so-called type-I or type-II coherence factors. ${ }^{18,19}$ Using the BCS solution for the Green's functions we find that for energies below the gap (for which there are no quasiparticles in the BCS limit), both $\alpha_{\text {TSTS }}^{\text {so }}$ and $\alpha_{\text {TSTS }}^{\mathrm{m}}$ vanish, and above 
the gap $\alpha_{\mathrm{TSTS}}^{\mathrm{so}}=1$ and $\alpha_{\mathrm{TSTS}}^{\mathrm{m}}=\left(E^{2}+\Delta^{2}\right) /\left(E^{2}-\Delta^{2}\right)>1$. Furthermore, we see from Eqs. (8b) and (8c) that for any $\theta(E)$, $\alpha_{\text {TSTS }}^{\mathrm{so}}<\alpha_{\text {TSTS }}^{\mathrm{m}}$. This implies that for a given normal state spin-flip length, the rate of spin flipping in the superconducting state is higher when the dominant spin-flip scattering mechanism is caused by magnetic impurities than if it is caused by spin-orbit interaction.

\section{MODEL}

We consider transport through an F/N/S/N/F hybrid wire ( $\mathrm{N}$ denotes a normal metal layer). It is assumed that the ferromagnets $(\mathrm{Co})$ are connected via normal metals $(\mathrm{Cu})$ to the superconductor $(\mathrm{Al})$. The distribution functions in the ferromagnet and the normal metal are determined by the Valet-Fert transport theory ${ }^{20}$ and in the superconductor by the theory described in the preceding section. An applied bias causes spin-polarized quasiparticles to be injected into the $\mathrm{S}$ layer. We assume that the magnetizations of the $\mathrm{F}$ parts are either parallel (P) or antiparallel (AP). Because of renormalized spin-flip rates and a reduction of the generalized spin-diffusion coefficient $\left(D_{\mathrm{L}}\right)$ in the superconductor, the magnetoresistance $\mathrm{MR} \equiv\left(R^{\mathrm{AP}}-R^{\mathrm{P}}\right) / R^{\mathrm{P}}$ is reduced for temperatures below $T_{c}$ compared to the normal metal state.

In order to determine the dominant contributions to the resistance of the system, we examine the magnitude of the resistance of the $\mathrm{F} / \mathrm{N}$ interface $\left(R_{\mathrm{F} / \mathrm{N}}\right)$ compared to the bulk resistance in $\mathrm{F}$ within a spin-flip length $\left(R_{\mathrm{sf}}^{\mathrm{F}}\right)$. The latter quantity is the largest resistance of the ferromagnet within its spin-active part. To this end, consider the ratio

$$
\frac{R_{\mathrm{F} / \mathrm{N}}}{R_{\mathrm{sf}}^{\mathrm{F}}}=\frac{A R_{\mathrm{F} / \mathrm{N}}}{\rho^{\mathrm{F}} l_{\mathrm{sf}}^{\mathrm{F}}} .
$$

We assume that $\mathrm{F}$ layers are made of $\mathrm{Co}, \mathrm{N}$ layers of $\mathrm{Cu}$, and the $\mathrm{S}$ layer of $\mathrm{Al}$. The bulk resistance of $\mathrm{Cu}$ as well as the proximity effect is neglected since the $\mathrm{Cu}$ layer is very thin, and in addition the typical resistivity of $\mathrm{Cu}$ is smaller than that of $\mathrm{Co}$ or $\mathrm{Al}$. The interface resistance $\left(A R_{\mathrm{F} / \mathrm{N}}\right)$, resistivity $\left(\rho^{\mathrm{F}}\right)$ and spin-diffusion length $\left(l_{\mathrm{sf}}^{\mathrm{F}}\right)$ for Co is reviewed in Ref. 21. It is found that $A R_{\mathrm{Cu} / \mathrm{Co}} \sim 0.5 \mathrm{f} \Omega \mathrm{m}^{2}$ at $4.2 \mathrm{~K}$ where $A$ is the cross section area. The renormalized resistivity ${ }^{20}$ is $\rho_{\text {Co }}^{*}$ $\sim 75 \mathrm{n} \Omega \mathrm{m}$, and $l_{\mathrm{sf}}^{\mathrm{Co}}=59 \mathrm{~nm}$ at $77 \mathrm{~K} .^{22}$ Thus we can conclude that for $\mathrm{Co} R_{\mathrm{F} / \mathrm{N}} / R_{\mathrm{sf}}^{\mathrm{F}} \approx 0.1$ as a least estimate since the spindiffusion length should be longer at $4.2 \mathrm{~K}$. This means that it is a valid approximation to disregard the interface resistances for long enough samples. The N/S interface resistance between $\mathrm{Cu}$ and $\mathrm{Nb}$ above the critical temperature is found to be larger than the $\mathrm{F} / \mathrm{N}$ resistance, ${ }^{23} A R_{\mathrm{Cu} / \mathrm{Nb}} \sim 1.10 \mathrm{f} \Omega \mathrm{m}^{2}$, and would give $R_{\mathrm{N} / \mathrm{s}} / R_{\mathrm{sf}}^{\mathrm{F}} \approx 0.2$. With $\mathrm{Al}$ as the superconducting layer we expect no higher interface resistance. We may also argue that the bulk resistance for dirty $\mathrm{Cu} / \mathrm{Co}$ layers scales as $A R_{\text {bulk }}^{\mathrm{Co}} \sim 0.1 L(\mathrm{~nm}) \mathrm{f} \Omega \mathrm{m}^{2},{ }^{24}$ where $L$ is the length of the layers expressed in $\mathrm{nm}$. Thus the bulk resistance for a slice of length $l_{\text {sf }}$ should be much larger than the interface resistance. Note that the resistance of a direct F/S interface is probably higher than the $\mathrm{F} / \mathrm{N} / \mathrm{S}$ structure in our model $\left(A R_{\mathrm{Nb} / \mathrm{Co}} \sim 3 \mathrm{f} \Omega \mathrm{m}^{2}\right.$, Ref. 21$)$, but since we include the $\mathrm{Cu}$ layers we can use only well-known parameters for F/S and
$\mathrm{N} / \mathrm{N}$ interface resistance in the above estimates.

The estimates above show that the interface resistances are much smaller than the relevant bulk resistances with the materials chosen here. The dominant contribution to the resistance and spin polarization of the current then comes from the bulk of F. We will later check that the change in resistance from normal to superconducting state is larger than the interface resistances. A possible approximation is therefore to neglect the interface resistances. This allows us to effectively do calculations for an F/S/F system with the boundary condition that the generalized diffusive current should be continuous which implies that the function $\theta$ is continuous at the interface. For strong ferromagnets the superconducting proximity effect into the ferromagnet is negligible and therefore $\theta=0$ in $\mathrm{F}$. Then we have by continuity $\theta \rightarrow 0$ in the superconductor close to the $\mathrm{F} / \mathrm{S}$ interfaces. This means, e.g., that the gap vanishes at the interface. In this case, it is the bulk transport properties that dominate the system, and there are no free parameters so that it is possible to give an unambiguous description of the transport properties. This is our aim in the rest of the paper.

The F/S/F system was motivated by the experiments of $\mathrm{Gu}$ et al. However, in those experiments Py was used for the ferromagnet, and because of the very short spin-diffusion length in this alloy $\left(l_{\mathrm{sf}}^{\mathrm{Py}}=5.5 \mathrm{~nm}\right)$ the interface resistances are of the same order as $R_{\mathrm{sf}}^{\mathrm{F}}$. Consequently, in these experiments both the interface resistances and the bulk resistance of $\mathrm{Al}$ are governed by superconductivity. Thus the model discussed above is not applicable, and the resistance of the spinpolarizing interface must be taken into account. To be specific, we no longer have that $\theta \rightarrow 0$ at the interfaces, and superconductivity is not completely suppressed at the interface as in the $\mathrm{Co} / \mathrm{Al} / \mathrm{Co}$ system. Using the approximations discussed above in calculations for the $\mathrm{Py} / \mathrm{Nb} / \mathrm{Py}$ system of Ref. 11 would therefore give a too low $T_{c}$. Numerical simulations and comparison with Ref. 11 show that this is indeed the case (not shown). A complete description of this experiment requires boundary conditions for the spin-polarizing interfaces given by scattering theory. This would describe the proximity effect in $\mathrm{N}$ as well as a reduction of the superconducting pairing amplitude close to the interface. However, as noted by Huertas-Hernando et al.,${ }^{25}$ this approach would require full knowledge about the interface scattering matrix, which is generally not available except for in simplified models at this moment.

\section{CAlCulations}

We have performed numerical calculations for a $\mathrm{Co} / \mathrm{Cu} / \mathrm{Al} / \mathrm{Cu} / \mathrm{Co}$ spin valve. Parameters for the superconductor are mostly taken from Ref. 26. The bulk value of the pairing potential at zero temperature is $\Delta_{0}=192 \mu \mathrm{eV}$ and the critical temperature $T_{c}=1.26 \mathrm{~K}$ with interaction parameter $N_{0} \lambda / 2=0.18 .{ }^{27}$ The normal state diffusion coefficient of $\mathrm{Al}$ is $D=160 \mathrm{~cm}^{2} / \mathrm{s}$, and the density of states at Fermi level $N_{0}$ $=2.2 \times 10^{47} \mathrm{~J}^{-1} \mathrm{~m}^{-3}$ corresponding to a resistivity $\rho_{\mathrm{Al}}^{\mathrm{N}}$ $=11 \mathrm{n} \Omega \mathrm{m}$. The normal state spin-flip relaxation length by spin-orbit interaction is given by the sample independent parameter $\varepsilon=l_{\mathrm{sf}} / l \approx 30,{ }^{28}$ and we assume that the elastic mean 


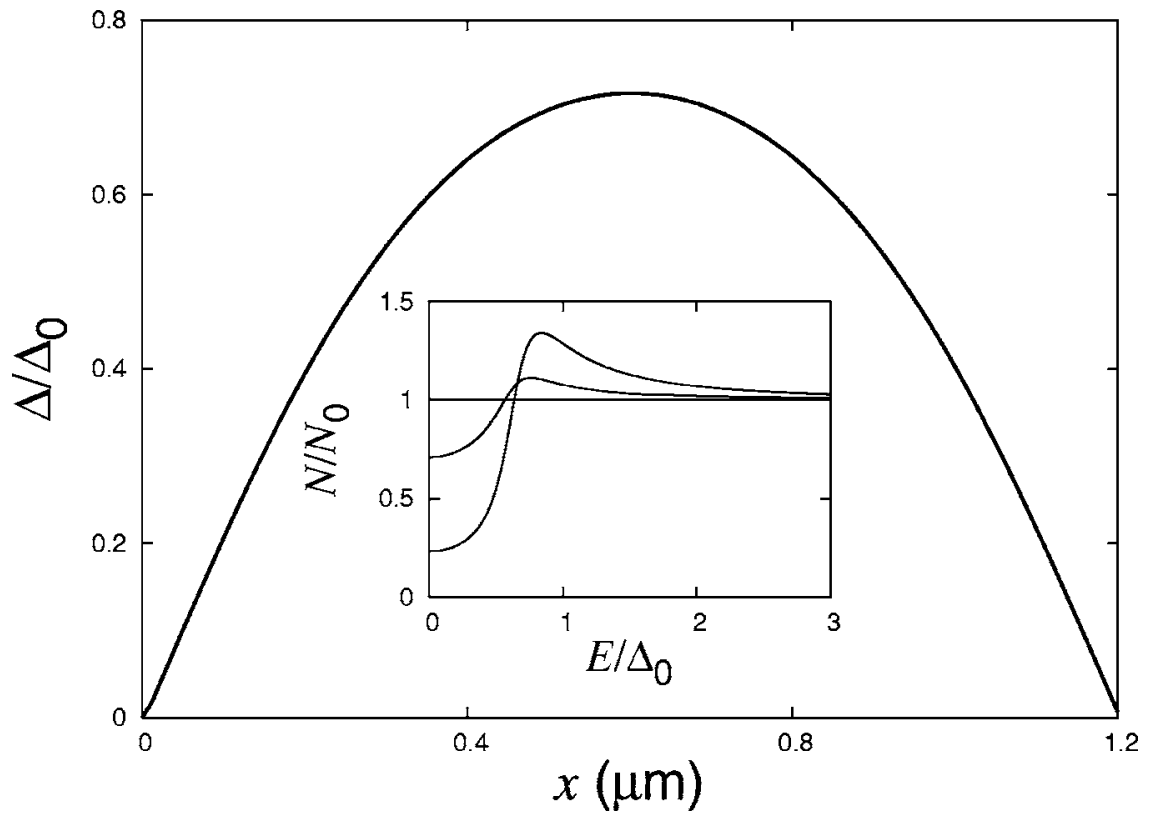

FIG. 1. Spatial variation of the pairing potential at $T / T_{c}=0.40$ scaled by $\Delta_{0}$. Inset, density of states at positions $0.0,0.2$, and $0.6 \mu \mathrm{m}$ into the $S$ wire. The curve evaluated at $x=0.0 \mu \mathrm{m}$ is identical to the normal state DOS (flat curve).

free path is $l=37 \mathrm{~nm}$. This gives $l_{\mathrm{sf}}=1.11 \mu \mathrm{m}$ for spin-orbit induced spin-flip. In calculations for magnetic impurity induced spin-flip we take the normal state value of the spin-flip length identical to the spin-orbit induced $l_{\text {sf }}$, but in general this length is determined by the impurity concentration which is sample specific. We take the length of the (identical) ferromagnetic elements to be $100 \mathrm{~nm}$ with a bulk spin asymmetry $\beta=0.4 .^{21}$ Figure 1 shows the spatial variation of the pairing potential resulting from complete suppression of superconductivity at the $\mathrm{F} / \mathrm{S}$ interfaces at reduced temperature $T / T_{c}=0.40$ for a $1.2 \mu \mathrm{m} \mathrm{Al}$ wire with magnetic impurities. The density of states at various locations in the superconductor is shown in the inset, and resembles the bulk BCS shape close to the center of the wire where the gap is largest.

A calculation of the resistance of the F/S/F system for parallel magnetizations is shown in Fig. 2. The $A R^{\mathrm{P}}$ values above the critical temperature agrees with analytical results

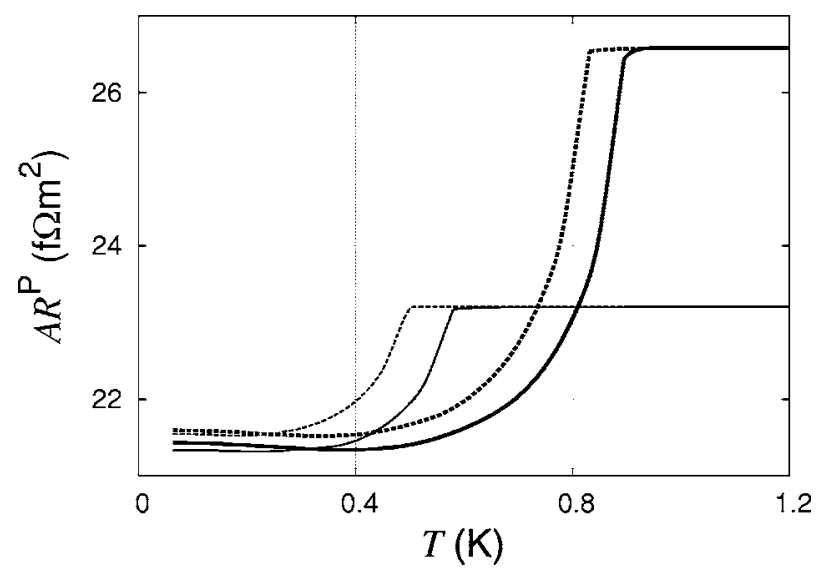

FIG. 2. Temperature dependence of resistance in parallel geometry for spin-orbit (solid lines) and magnetic impurity (dashed lines) induced spin flip. The thick curves are with Al length $1200 \mathrm{~nm}$, and the thin curves with $\mathrm{Al}$ length $900 \mathrm{~nm} . T_{c}$ is lowered by the presence of the magnetic impurities. based on the Valet-Fert theory. Below $T_{c}$ the resistance drops rapidly, but remains finite in the limit $T \rightarrow 0$. The change in resistance from normal to superconducting state is of the order of 2-6 $\mathrm{f} \Omega \mathrm{m}^{2}$ depending on the length of the superconductor, and this change is larger than the typical interface resistance, which should be checked as noted in Sec. III. The resistance of the system below $T_{c}$ is due to the $\mathrm{F}$ elements as well as the regions in the $\mathrm{S}$ wire next to the $\mathrm{F} / \mathrm{S}$ interface where there is conversion of current into supercurrent. ${ }^{26}$ The systems with magnetic impurities have the higher resistance as $T \rightarrow 0$, since the length of the resistive region near the interfaces is longer. This is because the conversion of current into supercurrent happens over a length scale determined by the coherence length $\xi=\sqrt{\hbar D / 2 \pi \Delta}$ which for a superconductor with magnetic impurities is longer since $\Delta$ is suppressed due to a term in the Usadel equation (9).

The dependence of the resistance on the magnetization configuration is shown in Fig. 3 where the excess resistance $\Delta R=A\left(R^{\mathrm{AP}}-R^{\mathrm{P}}\right)$ is plotted as a function of temperature. We show curves for systems with only spin-flip scattering from magnetic impurities or spin-orbit interaction. The systems with magnetic impurities provide a weaker suppression of the spin signal than systems with spin-orbit interaction. The opposite could be expected since as noted above $\alpha_{\text {TSTS }}^{\mathrm{m}}$ $>\alpha_{\text {TSTS }}^{\text {so }}$. However, the pairing potential is lower in a superconductor with magnetic impurities due to the detrimental effect of the impurities on superconductivity, and this is the dominant effect. This is confirmed by simulations of systems with equal strengths of the pairing potential, in which magnetic impurities is the strongest spin relaxation mechanism. From Fig. 3 we see that the difference in suppression of spin signal between spin-orbit and magnetic impurity induced spin-flip is smaller for the longer wires, since in this case the difference in $\Delta$ is also smaller. For long wires the excess resistance tends to zero at low temperatures as expected, because in this case the transport of spins through the superconductor is completely suppressed.

In Fig. 4 we show the spatial variation of the quasiparticle charge and spin current and supercurrent for the F/N/S /N/F 


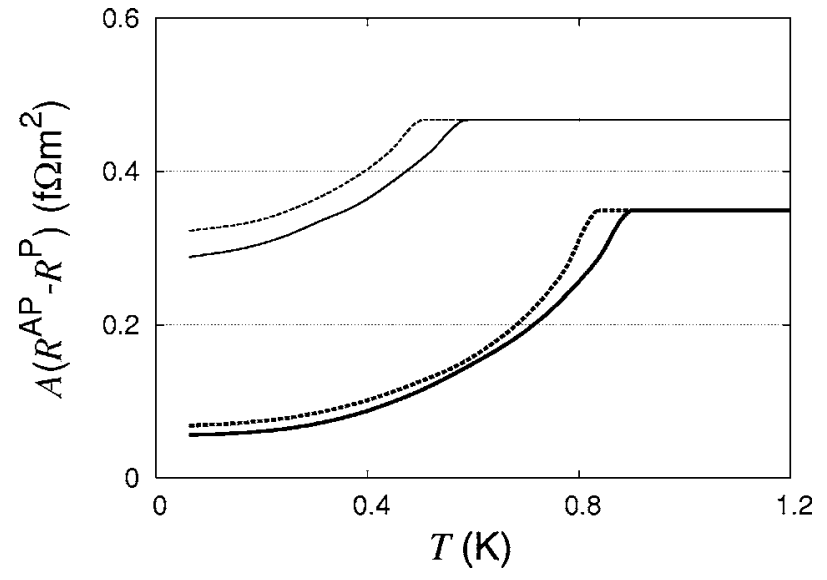

FIG. 3. Temperature dependence of the magnetoresistance for spin-orbit (solid lines) and magnetic impurity (dashed lines) induced spin flip. The top set of curves is a system with Al length $900 \mathrm{~nm}$, and the lower curves with Al length $1200 \mathrm{~nm}$.

spin valve with parallel magnetizations. The charge current is constant in the F parts of the wire, and is gradually converted into supercurrent in $\mathrm{S}$. Spin current injection into $\mathrm{S}$ is suppressed, as a comparison with the magnitude of spin current in the normal state shows. This leads to spin accumulation in $\mathrm{F}$ at the interfaces. We see that the spin current is reduced below $T_{c}$ inside the superconductor due to Cooper pairing. On the other hand, the total charge current increases below $T_{c}$ due to the reduced resistance of the superconductor. In Fig. 5 we show the spin accumulation $\mu_{\uparrow}-\mu_{\downarrow}$ for the same system. Comparison with the normal state shows that the spin accumulation is larger in the $\mathrm{S}$ case, due to the reduced penetration of spins into $S$ and since the net spin current out of the reservoirs is larger in the $\mathrm{S}$ case because the total resistance is lower. The spin accumulations that build up at the interfaces are relaxed through spin-flip in S. These spin

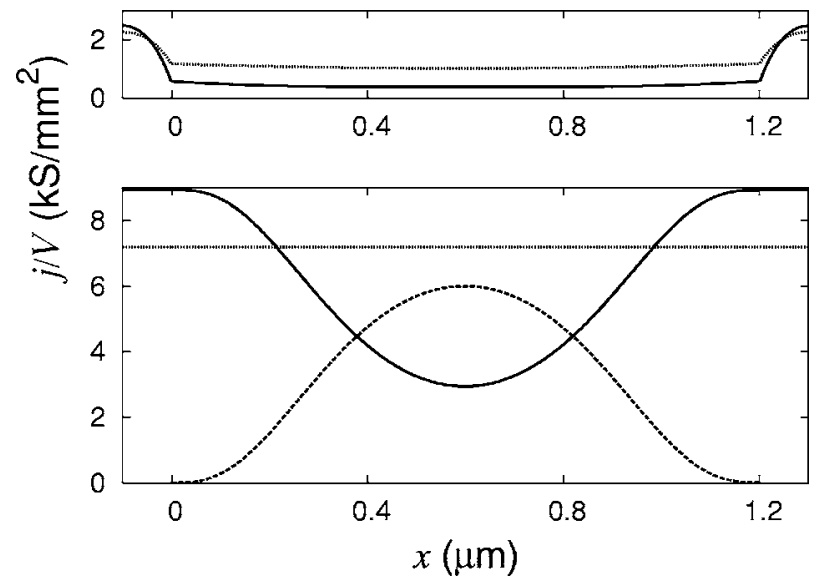

FIG. 4. Top panel, spatial dependence of the spin current for the F/S /F structure with Al length $1.2 \mu \mathrm{m}$ at $T / T_{c}=0.40$ (spin-orbit induced spin-flip). The normal state $\left(T>T_{c}\right)$ spin current is shown with the dotted line. The $F-S$ interfaces are at $x=0 \mu \mathrm{m}$ and $x$ $=1.2 \mu \mathrm{m}$. Bottom panel, spatial dependence of the quasiparticle charge current (solid line) and supercurrent (dashed line) for the same system as in the top panel. Normal state current is shown with dotted line.

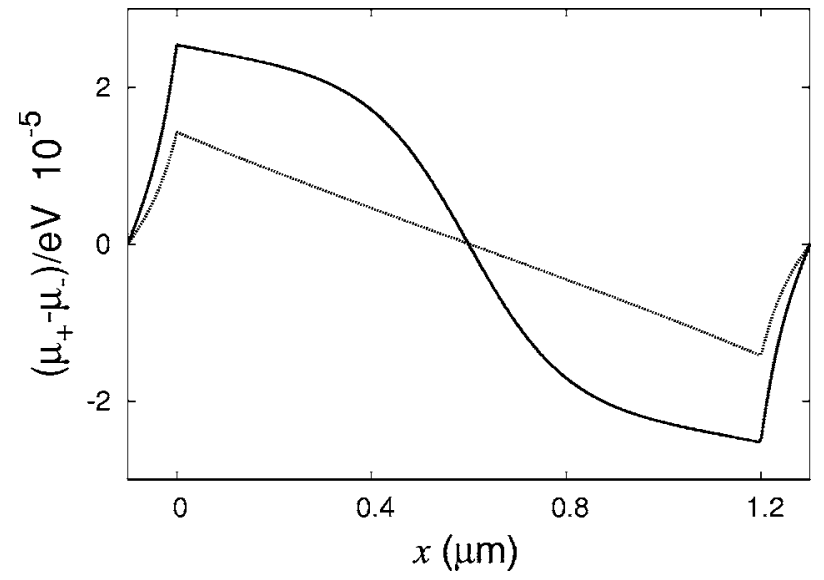

FIG. 5. The spatial dependence of the spin potential for the $\mathrm{F} / \mathrm{S} / \mathrm{F}$ structure with $\mathrm{Al}$ length $1.2 \mu \mathrm{m}$ at $T / T_{c}=0.40$ (solid line) and $T>T_{c}$ (dotted line) (spin-orbit induced spin flip). The F/S interfaces are at $x=0 \mu \mathrm{m}$ and $x=1.2 \mu \mathrm{m}$.

accumulations can be measured, e.g., by tunnel coupling between the superconductor and a third probe ferromagnet.

Qualitatively, our results for the MR are in agreement with the experiment by $\mathrm{Gu}$ et al. ${ }^{11}$ A contribution from the interfaces which is most probably important in the experiment, will not qualitatively change the properties of the system except for a higher $T_{c}$ as noted above. Therefore, quantitative differences between the experiment and our calculations using material parameters corresponding to the system in Ref. 11 are not surprising. A more detailed theoretical analysis, which accounts for interface resistance, should be made to enable a quantitative comparison with the experiments of Ref. 11, but this is beyond the scope of our present work. We emphasize again, that our predictions are, however, experimentally testable in $\mathrm{Co} / \mathrm{Al} / \mathrm{Co}$ spin valves, which can be fabricated using state-of-the-art technology.

\section{SUMMARY AND CONCLUSIONS}

In conclusion we have studied spin-transport properties of an $\mathrm{F} / \mathrm{S} / \mathrm{F}$ trilayer. We have developed transport equations using the quasiclassical theory of superconductivity and included the effects of spin-flip relaxation. An experimental system is proposed where interface resistance can be neglected and a simple description of the physics is possible. For this system we have performed numerical calculations of the magnetization-configuration dependent resistance. This demonstrates the dependence of the spin-transport suppression on different spin-flip mechanisms, i.e., magnetic impurities and spin-orbit interaction.

\section{ACKNOWLEDGMENTS}

This work was supported in part by The Research Council of Norway, NANOMAT Grants Nos. 158518/431 and 158547/431, RTN Spintronics, the Swiss NSF, the NCCR Nanoscience, and EU via Project No. NMP2-CT-2003505587 "SFINx." 
*Electronic address: jan.morten@ @hys.ntnu.no

${ }^{1}$ D. Beckmann, H. B. Weber, and H. v. Löhneysen, Phys. Rev. Lett. 93, 197003 (2004).

${ }^{2}$ B. Leridon, J. Lesueur, and M. Aprili, cond-mat/0411500 (unpublished).

${ }^{3}$ D. Huertas-Hernando, Y. V. Nazarov, and W. Belzig, Phys. Rev. Lett. 88, 047003 (2002).

${ }^{4}$ S. Oh and D. Youm, Appl. Phys. Lett. 71, 2376 (1997); L. R. Tagirov, Phys. Rev. Lett. 83, 2058 (1998); A. I. Buzdin, A. V. Vedyayev, and N. V. Ryzhanova, Europhys. Lett. 48, 686 (1999).

${ }^{5}$ V. T. Petrashov, I. A. Sosnin, I. Cox, A. Parsons, and C. Troadec, Phys. Rev. Lett. 83, 3281 (1999).

${ }^{6}$ M. Giroud, H. Courtois, K. Hasselbach, D. Mailly, and B. Pannetier, Phys. Rev. B 58, R11872 (1998).

${ }^{7}$ J. Aumentado and V. Chandrasekhar, Phys. Rev. B 64, 054505 (2001).

${ }^{8}$ F. J. Jedema, B. J. van Wees, B. H. Hoving, A. T. Filip, and T. M. Klapwijk, Phys. Rev. B 60, 16549 (1999).

${ }^{9}$ V. I. Fal'ko, C. J. Lambert, and A. F. Volkov, JETP Lett. 69, 532 (1999).

${ }^{10}$ W. Belzig, A. Brataas, Y. V. Nazarov, and G. E. W. Bauer, Phys. Rev. B 62, 9726 (2000).

${ }^{11}$ J. Y. Gu, J. A. Caballero, R. D. Slater, R. Loloee, and W. P. Pratt, Jr., Phys. Rev. B 66, 140507(R) (2002).

${ }^{12}$ T. Yamashita, S. Takahashi, H. Imamura, and S. Maekawa, Phys. Rev. B 65, 172509 (2002).

${ }^{13}$ J. P. Morten, A. Brataas, and W. Belzig, Phys. Rev. B 70, 212508 (2004).

${ }^{14}$ T. Yamashita, H. Imamura, S. Takahashi, and S. Maekawa, Phys.
Rev. B 67, 094515 (2003).

${ }^{15}$ A. Schmid, NATO ASI Ser., Ser. B 65, 423 (1981).

${ }^{16}$ A. Schmid and G. Schön, J. Low Temp. Phys. 20, 207 (1975).

${ }^{17}$ W. Belzig, F. K. Wilhelm, C. Bruder, G. Schön, and A. D. Zaikin, Superlattices Microstruct. 25, 1251 (1999).

${ }^{18}$ M. Tinkham, Introduction to Superconductivity (McGraw-Hill, New York, 1975).

${ }^{19}$ Y. Yafet, Phys. Lett. 98A, 287 (1983).

${ }^{20}$ T. Valet and A. Fert, Phys. Rev. B 48, 7099 (1993).

${ }^{21}$ J. Bass and W. P. Pratt, Jr., J. Magn. Magn. Mater. 200, 274 (1999).

${ }^{22}$ L. Piraux, S. Dubois, A. Fert, and L. Belliard, Eur. Phys. J. B 4, 413 (1998).

${ }^{23}$ W. Park, D. V. Baxter, S. Steenwyk, I. Moraru, W. P. Pratt, Jr., and J. Bass, Phys. Rev. B 62, 1178 (2000).

${ }^{24}$ A. A. Kovalev, A. Brataas, and G. E. W. Bauer, Phys. Rev. B 66, 224424 (2002).

${ }^{25}$ D. Huertas-Hernando, Y. V. Nazarov, and W. Belzig, cond-mat/ 0204116 (unpublished).

${ }^{26}$ G. R. Boogaard, A. H. Verbruggen, W. Belzig, and T. M. Klapwijk, Phys. Rev. B 69, 220503(R) (2004).

${ }^{27}$ H. Ibach and H. Lüth, Solid State Physics (Springer-Verlag, $\mathrm{GmbH}, 1995)$, p. 245, Table 10.1.

${ }^{28}$ F. J. Jedema, M. S. Nijboer, A. T. Filip, and B. J. van Wees, Phys. Rev. B 67, 085319 (2003).

${ }^{29}$ The quasiparticle charge current depends on the coordinate $x$, but conservation of charge is satisfied when including the supercurrent contribution so that the total charge current does not depend on $x$. 